\title{
Chemical abundances in star-forming galaxies and damped Lyman alpha systems
}

\author{
Regina E. Schulte-Ladbeck, Brigitte König and Brian Cherinka
}

Department of Physics and Astronomy, University of Pittsburgh, Pittsburgh, PA 15260, USA

\begin{abstract}
We investigate the chemical abundances of local star-forming galaxies which cause damped Lyman alpha lines. A metallicity versus redshift diagram was constructed, on which the chemical abundances of low-redshift star-forming galaxy populations are compared with those of high-redshift damped Lyman alpha systems. We discuss two types of experiments on individual star-forming galaxies. In the first, the damped Lyman alpha line is created against an internal ultraviolet light source generated by a star-forming cluster or a supernova explosion. In the second, the damped Lyman alpha line is seen against a background quasar. The metallicities measured from ionised gas in the star-forming regions, and neutral gas in the damped Lyman alpha systems, are compared with one another on a case-by-case basis. We highlight the occurrence of the star-forming galaxy/quasar pair SBS 1543+593/HS 1543+5921, where the emission- and absorption-line derived abundances give the same result. We argue that we therefore can in principle interpret damped Lyman alpha system metallicities as an extension of star-forming galaxy metallicities to higher redshifts, supporting the idea that gas-rich galaxies had lower chemical abundances when they were younger.
\end{abstract}

\section{Introduction}

The chemical evolution of the Universe occurs via two channels. Big-bang nucleosynthesis generated several light chemical elements during "the first three minutes". In the following $13 \mathrm{Gyr}$, metals were synthesised by stars in galaxies, a process which continues until the present epoch. The ratios of certain metals, for example, those of the $\alpha$-to-irongroup elements, can be used to constrain the evolution of galaxies.

Gas-phase chemical abundances in the high-redshift Universe have been obtained by the study of QSO absorption lines, and traditionally refer to iron-peak elements (e.g. Prochaska et al. 2003). It is generally thought that absorbers with high neutral hydrogen column densities, such as damped Lyman alpha (DLA) systems and sub-DLAs, originate in gas-rich galaxies. Typical impact parameters needed to reach the DLA-defining column density $\mathrm{N}$ of $2 \times 10^{20} \mathrm{~cm}^{-2}$ are expected to be of the order of a few $10 \mathrm{~s}$ of $\mathrm{kpc}$, based on the HI sizes of local gas-rich galaxies (Rosenberg \& Schneider 2003). However, the connection between DLAs and galaxies is far from clear. For instance, the very low metallicity of high-redshift DLAs has been interpreted to indicate that DLA galaxies are preferentially dwarf galaxies, in which case they would not be representative of the local field galaxy population (e.g. Kulkarni et al. 2005).

Only at low redshifts can the galaxies responsible for causing DLAs be observed directly. We discuss here a few cases for which detailed studies are in progress. Chemical properties of galaxies at low redshifts are based on measurements of emission lines from photo-ionised gas, and refer to the $\alpha$-capture element oxygen (e.g. Garnett 2004). Fig. 1 illustrates the local $\alpha$-element abundances derived from emission-lines, and the high-redshift abundances derived for DLAs from absorption lines, showing only those for which $\alpha$ - rather than Fe-peak element abundances are available. We also indicate 
the maximum and minimum oxygen abundances seen in the local Universe, and note that a) there are no local galaxies known with abundances as low as those of the lowestmetallicity DLAs; and b) there are no DLAs known which reach super-Solar abundances known locally to occur in the most luminous SFGs. The chemical abundances of Lyman Break Galaxies (LBGs) and of DLAs are beginning to show some overlap at a redshift of about 2, where emission-line abundances of LBGs have recently become available (e.g. Teplitz et al. 2000; Shapley et al. 2004). LBGs are luminous star-forming galaxies (SFG), which are presumed to be offset from the local luminosity-metallicity relation because of their youth $\dagger$.

In order to compare the properties of SFGs with those of high-redshift DLAs, we need to answer the question whether emission- and absorption-line techniques yield concordant values for chemical abundances. The experiment requires the use of a SFG, a background light source, and a high neutral H column density on its path. For some time, the neutral gas in SFGs has been probed against internal star clusters. More recently, the studies have been expanded to bona fide DLA galaxies using SFG-QSO pairs.

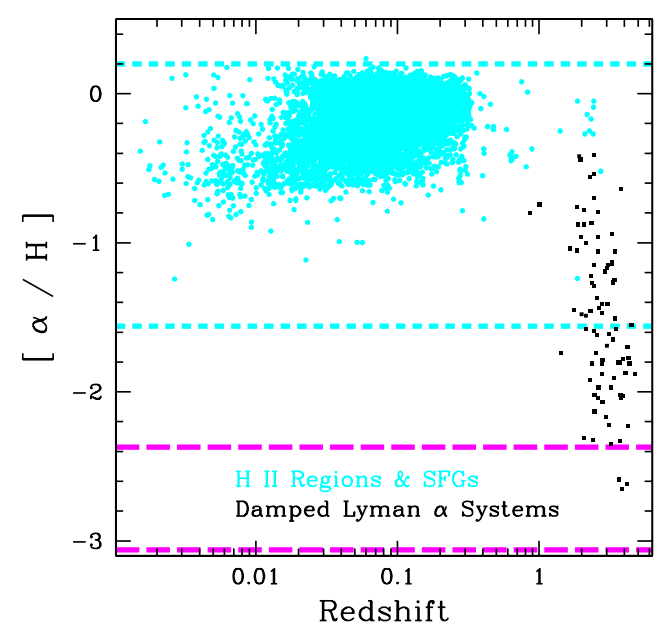

Figure 1. Metallicity as a function of redshift. The bright circles are values of $[\mathrm{O} / \mathrm{H}]_{\mathrm{II}}$ for HII regions and SFGs from the SDSS. These are extended to redshifts above 0.3 by using data from Shapley et al. (2004), Steidel et al. (2004), Koo et al. (1994), Kobulnicky \& Zaritsky (1999), Lemoine-Busserolle et al. (2003), Liang et al. (2004), Rigopoulou et al. (2000), Maier et al. (2004), Contini et al. (2002), Cardiel et al. (2003), and Teplitz et al. (2000). The black squares are values of $[\mathrm{O}, \mathrm{S}, \mathrm{Si} / \mathrm{H}]_{\mathrm{I}}$ from the compilation of Prochaska et al. (2003). The short-dashed lines show the maximum and minimum values of oxygen abundances in local HII regions and SFGs based on the direct or $\mathrm{T}_{e}$ method (Izotov \& Thuan 1999; Castellanos, Díaz \& Terlevich 2002). The long-dashed lines are the theoretical expectations of oxygen enrichment from the first supernovae (Salvaterra \& Ferrara 2003), assuming a non-rotating (lower line) and a rotating (upper line) Population III progenitor.

\section{SFGs causing DLAs in their own spectra}

Table 1 provides a list of local SFGs exhibiting DLA lines in their own UV spectra. Chemical abundances are referenced to the Solar values of Holweger (2001). For oxygen this is $12+\log \mathrm{O} / \mathrm{H}=8.736 \pm 0.078$. The neutral gas-phase abundances are for oxygen, with the exception of MS 1512-cB58, where they refer to the average of several $\alpha$ elements. Oxygen abundances in the ionised gas phase are given as well. We illustrate how the abundances of these SFGs compare to other low-redshift SFGs and to high-redshift DLAs in Fig. 2.

$\dagger$ We use a cosmology with $\mathrm{H}_{0}=70 \mathrm{~km} \mathrm{~s}^{-1} \mathrm{Mpc}^{-1}, \Omega_{m}=0.3$, and $\Omega_{\Lambda}=0.7$. All literature data were converted to this cosmology when needed. 

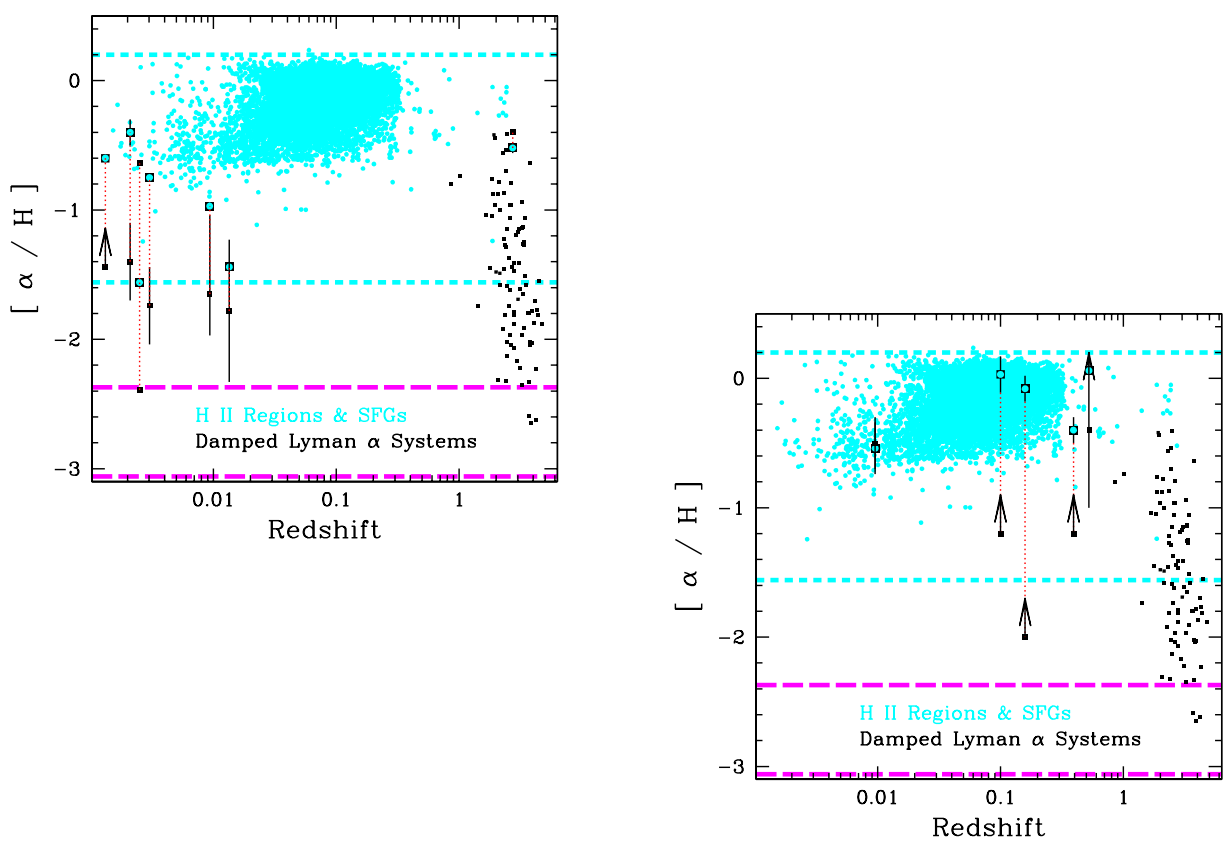

Figure 2. Left - Metallicity as a function of redshift as in Fig. 1. Over-plotted are the abundances of SFGs from Table 1, which display DLAs in their own spectra. For each SFG which is also a DLA, the bright symbols mark the ionised gas abundance, and they are connected by dotted lines, to dark squares which indicate the neutral gas abundance. For I $\mathrm{Z}_{\mathrm{w}} 18$, the extrema of neutral gas metallicities allowed by the analyses of two teams are shown.

Figure 3. Right - Over-plotted here are the abundances of SFGs from Table 2, and the corresponding unambiguous abundances (or abundance limits) determined on the sight-line to the QSO.

The advantage of examining DLAs produced within SFGs by their own starburst clusters, is that the neutral and the ionised gas are probed on the exact same sightline. A disadvantage of this technique is that the background sources are extended. The sight-line also traverses some ionised gas.

So far, six out of seven SFGs studied with this technique are dwarf galaxies with small star-formation rates $(\mathrm{SFR})$. In general in these galaxies $[\mathrm{O} / \mathrm{H}]_{\mathrm{II}}-[\mathrm{O} / \mathrm{H}]_{\mathrm{I}}>0$ (although the case of I Zw 18 is controversial). The seventh galaxy studied is the luminous, gravitationally-lensed, high-redshift LBG MS 1512-cB58. Here, $[\mathrm{O} / \mathrm{H}]_{\mathrm{II}}$ and $[\mathrm{O} / \mathrm{H}]_{\mathrm{I}}$ are in close agreement.

The discovery of DLA lines in the spectra of gamma ray burst (GRB) source afterglows has recently opened up a new window on neutral gas metallicities at high redshift (e.g. Vreeswijk et al. 2004). GRBs are thought to originate from supernova explosions in starforming regions, and indeed some GRBs exhibit Lyman $\alpha$ emission lines in their DLA troughs. At this time, abundance analyses of the ionised gas in GRB-DLAs are not available, thus none of these objects are listed in Table 1.

\section{SFGs causing DLAs in the spectra of background QSOs}

Schulte-Ladbeck et al. (2005) recently studied the $\alpha$-element abundances of the DLA galaxy SBS $1543+593$ in emission and absorption. The background QSO HS 1543+5921 
Table 1. SFGs causing Lyman alpha lines in their own spectra

\begin{tabular}{|c|c|c|c|c|c|c|c|c|}
\hline Galaxy & $\mathrm{z}$ & $\log \left(\mathrm{N}_{\mathrm{HI}}\right)$ & Type & $\mathrm{M}_{B}$ & $\begin{array}{c}\mathrm{L} \\
{\left[L^{*}\right]}\end{array}$ & $\frac{\mathrm{SFR}}{\left[\mathrm{M}_{\odot} \mathrm{yr}^{-1}\right]}$ & {$[\mathrm{O} / \mathrm{H}]_{\mathrm{II}}$} & {$[\mathrm{O} / \mathrm{H}]_{\mathrm{I}}$} \\
\hline NGC 625 & 0.001321 & 20.5 & DIRR & -16.3 & 0.03 & 0.05 & $-0.6^{12}$ & $>-1.4^{11}$ \\
\hline NGC 1705 & 0.00210 & 20.20 & $\mathrm{BCD}$ & -15.8 & 0.02 & 0.03 & $-0.4^{5}$ & $-1.4^{4}$ \\
\hline I $\mathrm{Zw} 18$ & 0.00250 & 21.34 & $\mathrm{BCD}$ & -14.3 & 0.004 & 0.09 & $-1.56^{1}$ & $-1.4^{6},-2.1^{7}$ \\
\hline Mrk 59 & 0.003631 & 20.8 & $\mathrm{BCD}$ & -17.5 & 0.08 & 1.5 & $-0.75^{1}$ & $-1.7^{8}$ \\
\hline I $\mathrm{Zw} 36$ & 0.0094 & 21.30 & $\mathrm{BCD}$ & $<-13.9$ & $>0.003$ & $>0.03$ & $-0.97^{1}$ & $-1.7^{9}$ \\
\hline SBS0335-052 & 0.013486 & 21.85 & $\mathrm{BCD}$ & -17.1 & 0.04 & 1.3 & $-1.44^{1}$ & $-1.8^{10}$ \\
\hline MS1512-cB58 & 2.7276 & 20.85 & $\mathrm{LBG}$ & -22.0 & 5.3 & $>100$ & $-0.52^{2}$ & $-0.4^{3}$ \\
\hline
\end{tabular}

Table 2. SFGs causing Lyman alpha lines in background QSO spectra

\begin{tabular}{|c|c|c|c|c|c|c|c|c|c|c|c|}
\hline QSO & $\begin{array}{c}\mathrm{b} \\
{[\mathrm{kpc}]}\end{array}$ & Galaxy & $\mathrm{z}$ & $\log \left(N_{\mathrm{HI}}\right)$ & Type & $\mathrm{M}_{B}$ & $\begin{array}{c}\mathrm{L} \\
{\left[L^{*}\right]}\end{array}$ & $\begin{array}{c}\mathrm{SFR} \\
{\left[\mathrm{M}_{\odot} \mathrm{yr}^{-1}\right]}\end{array}$ & {$[\mathrm{O} / \mathrm{H}]_{\mathrm{II}}$} & {$[\mathrm{O} / \mathrm{H}]_{\mathrm{I}}$} & $\begin{array}{l}\alpha, \text { other } \\
\text { than } \mathrm{O}\end{array}$ \\
\hline HS $1543+5921$ & 0.5 & SBS $1543+593$ & 0.0096 & 20.3 & $\mathrm{Sm}$ & -16.8 & 0.04 & 0.006 & $-0.59^{a}$ & $-0.5^{*}$ & $\mathrm{~S}, \mathrm{Si}$ \\
\hline $\mathrm{Q} 1543+489$ & 107 & Galaxy 5 & 0.0382 & 18.4 & $\mathrm{~S}$ & -17.9 & 0.12 & 0.37 & $-0.06^{f}$ & $>-0.8 ?$ & $\mathrm{~S}, \mathrm{Mg}$ \\
\hline PHL 909 & 140 & SDSS J005719+ & 0.0822 & $\leqslant 17.7$ & $\mathrm{Sc}$ & -19.7 & 0.6 & 1.5 & $-0.22^{g}$ & $\ldots$ & \\
\hline $\begin{array}{l}\text { PKS } 0439-433 \\
\text { PHL } 909\end{array}$ & $\begin{array}{c}7 \\
19\end{array}$ & $\begin{array}{r}\text { Object } 1 \\
\text { SDSS J005710 }\end{array}$ & $\begin{array}{l}0.1012 \\
0.1237\end{array}$ & $\begin{array}{c}19.7 \\
18.58\end{array}$ & $\begin{array}{l}\mathrm{Sab} \\
\mathrm{Sa}\end{array}$ & $\begin{array}{l}-20.2 \\
-18.5\end{array}$ & $\begin{array}{l}1.0 \\
0.2\end{array}$ & $\begin{array}{l}0.4 \\
1.1\end{array}$ & $\begin{array}{c}0.03^{b} \\
-0.15^{g}\end{array}$ & $\begin{array}{l}>-1.2 \\
>-1.2 ?\end{array}$ & $\mathrm{Mg}, \mathrm{Si}$ \\
\hline $\begin{array}{l}\text { PHL } 1226 \\
\text { Q1209+107 }\end{array}$ & $\begin{array}{c}17.6 \\
38\end{array}$ & $\begin{array}{r}\text { G4 } \\
\text { Galaxy } 19 / 8\end{array}$ & $\begin{array}{l}0.1602 \\
0.3930\end{array}$ & $\begin{array}{c}19.7 \\
19.54\end{array}$ & $\begin{array}{c}S \\
\text { Int }\end{array}$ & $\begin{array}{l}-20.0 \\
-18.5\end{array}$ & $\begin{array}{l}0.8 \\
0.2\end{array}$ & $\begin{array}{l}0.5 \\
1.7\end{array}$ & $\begin{array}{l}-0.08^{d} \\
-0.4^{c, e}\end{array}$ & $\begin{array}{l}>-2.0 \\
>-1.2\end{array}$ & \\
\hline B $20827+243$ & 36 & G1 & 0.525 & 20.3 & $\mathrm{~S}$ & -19.8 & 0.69 & 0.08 & $>0.06^{b}$ & $>-2.8$ & $\mathrm{~S}, \mathrm{Si}$ \\
\hline
\end{tabular}

${ }^{a}$ Schulte-Ladbeck et al. (2005), ${ }^{b}$ Chen et al. (2005), ${ }^{c}$ Cristiani (1987), ${ }^{d}$ Christensen et al. (2005)

$e$ Yanny (1990), $f$ Bowen et al. (1997), $g$ this work

The $[\mathrm{O} / \mathrm{H}]_{\mathrm{I}}$ values were derived assuming the optically thin case and applying the approximation formula from

Petitjean (1998). * [S/H], + also SDSS J154519

has an impact parameter of $2.4^{\prime \prime}$, and intercepts the dwarf galaxy's disk close to its centre. SBS $1543+593$ offers an excellent opportunity to directly compare element abundances inferred from cool interstellar gas (DLAs) and ionised gas (SFGs). None of the previously imaged DLA galaxies are resolved enough to show individual HII regions. In none of them does the sight-line to the QSO intercept the disk of the galaxy close to its centre, eliminating concerns over disk metallicity gradients.

The brightest HII region in SBS $1543+593$ yields $[\mathrm{O} / \mathrm{H}]_{\mathrm{II}}=-0.54 \pm 0.20$ and $[\mathrm{S} / \mathrm{H}]_{\mathrm{II}}=$ $-0.27 \pm 0.30$ (where the Solar S abundance $(7.20 \pm 0.06)$ is referenced to Grevesse \& Sauval 1998). HST/FOS data reveal $[\mathrm{O} / \mathrm{H}]_{\mathrm{I}}>-2.14$, and $\mathrm{HST} / \mathrm{STIS}$ data give $[\mathrm{S} / \mathrm{H}]_{\mathrm{I}}=-0.50 \pm$ 0.33 . Within the errors these four values are the same. Also, log $(\mathrm{N} / \mathrm{O})_{\mathrm{II}}=-1.40_{-0.30}^{+0.20}$ is within the range of $-2.0 \lesssim \log (\mathrm{N} / \mathrm{S})_{\mathrm{I}} \lesssim-0.8$, suggesting agreement.

In Fig. 3 we show $[\mathrm{S} / \mathrm{H}]_{\mathrm{I}}$ for the neutral-gas abundance, and $[\mathrm{O} / \mathrm{H}]_{\mathrm{II}}$ for the ionised-gas abundance. Here is one DLA for which we can demonstrate that in principle, emissionand absorption-line techniques give the same results when chemical elements with similar nucleosynthetic origins are compared at similar locations within a DLA galaxy. This result validates in principle comparisons between the two types of objects, SFGs and DLAs, on the metallicity versus redshift diagram. SBS $1543+593$ thus supports the case that metal enrichment has taken place in the gas-rich star-forming galaxy population between redshifts of 5 and 0 . In practise, galaxy metallicity gradients will be important for a detailed comparison between SFG and DLA metallicities (see e.g. Christensen et al. 2005; Chen et al. 2005).

Table 2 summarises the result for a few additional SFG/QSO pairs, for which we were able to list $\alpha$-element abundances in the ionised phase, and derive abundance limits in 
the neutral phase. We find that in two sub-DLAs (G4/PHL 1226 and Object1/PKS 0439$433)$, the QSO intercepts the disk just at its outer radius. Here $[\alpha / \mathrm{H}]_{\mathrm{I}}$ is consistent with expectations of the local disk metallicity gradient of Ferguson et al. (1998). As the impact parameter grows, this extrapolation must eventually become invalid. We note that the present sample traces $\alpha$ elements to a distance of about $100 \mathrm{kpc}$ from SFGs.

Chen et al. (2005) have argued that low-redshift DLAs can be explained via a combination of gas cross section selection and metallicity gradients. Galaxy metallicity gradients have only been investigated locally. We do not have any insight into how they may evolve with redshift. One point in particular is worth pondering. It is now becoming evident that the optical sizes of galaxies grow smaller with increasing redshift (Bouwens et al. 2004). This might suggest an as yet little explored bias in the observed metallicity-redshift dependence of DLAs.

\section{Conclusions}

- Emission and absorption diagnostics of one SFG/QSO pair giving rise to a DLA are shown to give the same $\alpha$-element abundances for the ionised gas in the SFG and the neutral gas in the DLA.

- Emission- and absorption-derived abundance offsets in SFG/QSO pairs in which the impact parameter is less than or equal to the optical radius of the galaxy so far appear consistent with the expectation of a disk metallicity gradient.

- High-redshift SFGs and DLAs exhibit lower abundances than their low-redshift counterparts, indicating that gas-rich galaxies had lower abundances when they were younger.

\section{Acknowledgements}

This paper makes use of publicly available SDSS data. The SDSS website is http:// www.sdss.org/. Chris Miller is thanked for providing the catalogue of line fluxes from which the SDSS HII-region and SFG metallicities were derived. This paper was funded in part by NASA HST archival project 10282. We thank the School of Arts \& Sciences for support.

\section{References}

Aloisi, A., Savaglio, S., Heckman, T. M., Hoopes, C. G., Leitherer, C., Sembach, K. R., 2003, ApJ, 595, 760

Bouwens, R. J., Illingworth, G. D., Blakeslee, J. P., Broadhurst, T. J., Franx, M., 2004, ApJ, $611, \mathrm{~L} 1$

Bowen, D. V., Osmer, S. J., Blades, J. C., Tytler, D., 1997, MNRAS, 284, 599

Cannon, J. M., Skillman, E. D., Sembach, K. R., Bomans, D. J., 2005, ApJ, 618, 247

Cardiel, N., Elbaz, D., Schiavon, R. P., Willmer, C. N. A., Koo, D. C., Phillips, A. C., Gallego, J., 2003, ApJ, 584, 76

Castellanos, M., Díaz, A. I., Terlevich, E., 2002, MNRAS, 329, 315

Chen, H.-W., Kennicutt Jr, R. C., Rauch M., 2005, astro-ph/0411006

Christensen, L., Schulte-Ladbeck, R. E., Sánchez, S. F., Becker, T., Jahnke, K., Kelz, A., Roth, M. M., Wisotzki, L., 2005, A\&A, 429, 477

Cristiani, S., 1987, A\&A, 175, L1

Contini, T., Treyer, M. A., Sullivan, M., Ellis, R. S., 2002, MNRAS, 330, 75

Ferguson, A. M. N., Gallagher, J. S., Wyse, R. F. G., 1998, AJ, 116, 673

Garnett, D. R., 2004, Cosmochemistry. The melting pot of the elements, 171

Grevesse, N., Sauval, A. J., 1998, Space Science Reviews, 85, 161 
Heckman, T. M., Sembach, K. R., Meurer, G. R., Strickland, D. K., Martin, C. L., Calzetti, D., Leitherer, C., 2001, ApJ, 554, 1021

Holweger, H., 2001, AIP Conf. Proc. 598: Joint SOHO/ACE workshop "Solar and Galactic Composition", 598, 23

Izotov, Y. I., Thuan, T. X., 1999, ApJ, 511, 639

Izotov, Y. I., Thuan, T. X., 2002, ApJ, 567, 875

Kobulnicky, H. A., Zaritsky, D., 1999, ApJ, 511, 118

Koo, D. C., Bershady, M. A., Wirth, G. D., Stanford, S. A., Majewski, S. R., 1994, ApJ, 427, L9

Kulkarni, V. P., Fall, S. M., Lauroesch, J. T., York, D. G., Welty, D. E., Khare, P., Truran, J. W., 2005, ApJ, 618, 68

Lebouteiller, V., Kunth, D., Lequeux, J., Lecavelier des Etangs, A., Désert, J.-M., Hébrard, G., Vidal-Madjar, A., 2004, A\&A, 415, 55

Lecavelier des Etangs, A., Désert, J.-M., Kunth, D., Vidal-Madjar, A., Callejo, G., Ferlet, R., Hébrard, G., Lebouteiller, V., 2004, A\&A, 413, 131

Lemoine-Busserolle, M., Contini, T., Pelló, R., Le Borgne, J.-F., Kneib, J.-P., Lidman, C., 2003, A\&A, 397, 839

Liang, Y. C., Hammer, F., Flores, H., Elbaz, D., Marcillac, D., Cesarsky, C. J., 2004, A\&A, 423, 867

Maier, C., Meisenheimer, K., Hippelein, H., 2004, A\&A, 418, 475

Petitjean, P., 1998, astro-ph/9810418

Pettini, M., Rix, S. A., Steidel, C. C., Adelberger, K. L., Hunt, M. P., Shapley, A. E., 2002, ApJ, 569, 742

Prochaska, J. X., Gawiser, E., Wolfe, A. M., Castro, S., Djorgovski, S. G., 2003, ApJ, 595, L9

Rigopoulou, D., et al., 2000, ApJ, 537, L85

Rosenberg, J. L., Schneider, S. E., 2003, ApJ, 585,

Salvaterra, R., Ferrara, A., 2003, MNRAS, 340, L17

Schulte-Ladbeck, R. E., König, B., Miller, C. J., Hopkins, A. M., Drozdovsky, I. O., Turnshek, D. A., Hopp, U., submitted to ApJL 256

Schulte-Ladbeck, R. E., Rao, S. M., Drozdovsky, I. O., Turnshek, D. A., Nestor, D. B., Pettini, M., 2004, ApJ, 600, 613

Shapley, A. E., Erb, D. K., Pettini, M., Steidel, C. C., Adelberger, K. L., 2004, ApJ, 612, 108

Skillman, E. D., Côté, S., Miller, B. W., 2003, AJ, 125, 610

Steidel, C. C., Shapley, A. E., Pettini, M., Adelberger, K. L., Erb, D. K., Reddy, N. A., Hunt, M. P., 2004, ApJ, 604, 534

Storchi-Bergmann, T., Calzetti, D., Kinney, A. L., 1994, ApJ, 429, 572

Teplitz, H. I., et al., 2000, ApJ, 533, L65

Thuan, T. X., Lecavelier des Etangs, A., Izotov, Y. I., 2005, ApJ, 621, 269

Vreeswijk, P. M., et al., 2004, A\&A, 419, 927

Yanny, B., 1990, ApJ, 351, 396 